\title{
AUTHOR REPLY-An Unusual Case of Cardiac Amyloidosis
}

To the Editors:-We agree with Dr. Bucciarelli-Ducci that cardiovascular magnetic resonance (CMR) is a promising diagnostic test in patients with suspected cardiac involvement from systemic amyloidosis. Maceira et al. ${ }^{1}$ in their study of 29 patients with presumed cardiac amyloidosis found a characteristic pattern of global subendocardial enhancement of the left ventricle that was distinct from control patients with hypertensive cardiomyopathy. The study did not compare CMR to echocardiography to establish the diagnosis of cardiac amyloidosis but did suggest that CMR might provide a viable alternative noninvasive strategy for identifying cardiac amyloid patients. Perugini et al. ${ }^{2}$ performed a prospective analysis of 21 patients with biopsy proven $\mathrm{AL}$ amyloidosis or familial amyloidosis and found that 16 of their patients (76\%) had abnormal gadolinium enhancement, although the distribution was more heterogeneous. It is interesting to note that the five patients without gadolinium enhancement all had both echocardiographic and histologic evidence of cardiac amyloidosis, suggesting that some patients will be missed if CMR is the only diagnostic test performed.

Dr. Bucciarelli-Ducci et al. ${ }^{3}$ have elegantly demonstrated how CMR findings coupled with echocardiography and other clinical features of amyloidosis may obviate the need for endomyocardial biopsy in select patients. CMR may be able to detect patients with early myocardial involvement before they develop echocardiographic findings or clinical symptoms, although this has yet to be formally studied. ${ }^{4}$ It has also been suggested that CMR may be a useful noninvasive means to follow cardiac involvement in amyloid patients after treatment because echocardiography is limited in this regard. ${ }^{1}$ Dr. Bucciarelli-Ducci's suggestion of using CMR to target areas for endomyocardial biopsy is also an intriguing application of this technique.

As Dr. Bucciarelli-Ducci points out, CMR is not available at all centers as it requires experienced personnel to both administer and interpret the test. While its role has yet to be clearly defined, CMR is likely to become an important part of the armamentarium in the diagnosis and management of cardiac amyloidosis and should be considered as an adjunctive noninvasive test in patients with suspected amyloid myocardial deposition.

Brian Garibaldi, and David Zaas, MD, Department of Medicine, The Johns Hopkins Hospital, Baltimore, MD, USA; (e-mail: bgariba1@ jhmi.edu)

\section{REFERENCES}

1. Maceira AM, Joshi J, Prasad SK, et al. Cardiovascular magnetic resonance in cardiac amyloidosis. Circulation. 2005;111:122-4.

2. Perugini E, Rapezzi C, Piva T, et al. Non-invasive evaluation of the myocardial substrate of cardiac amyloidosis by gadolinium cardiac magnetic resonance. Heart. 2006;92:343-9.

3. Bucciarelli-Ducci C, Locca D, Barbeau G, Prasad SK. Value of cardiovascular magnetic resonance for determining cardiac involvement in systemic amyloidosis. Eur Heart J. 2007;28:1186.

4. Kwong RY, Falk RH. Cardiovascular magnetic resonance in cardiac amyloidosis. Circulation. 2005;111(2):122-4.

(ㄷ) 2007 Society of General Internal Medicine 2007;22:1383

DOI: $10.1007 / \mathrm{s} 11606-007-0287-0$

Published online July 20, 2007 